\title{
Finiteness of Numbers of Curves on a Minimal Surface with $\kappa=1$
}

By

\author{
Yoshio Fujimoto
}

\section{$\S 0$. Introduction}

In [4], Namikawa proposed the following problem.

Problem. Let $S$ be a minimal compact analytic surface. For an arbitrary curve (i.e. a reduced, irreducible effective divisor) $C$ on $S$, let us define the arithmetic genus of $C$ by

$$
\pi(C)=\frac{1}{2} C\left(C+K_{S}\right)+1, \quad \text { where } K_{S} \text { is the canonical divisor on } S .
$$

If we fix a non-negative integer $g$, how many algebraic families of curves of genus $g$ are there on $S$ ?

And he proved the finiteness of numbers of algebraic families of curves with the fixed arithmetic genus modulo $\operatorname{Aut}(S)$ except the case where $\kappa(S)=1$. The purpose of this note is to prove that the above finiteness also holds in the case where $\kappa(S)=1$. We state our main theorem.

Theorem A. Let $S$ be a minimal analytic surface with $\kappa(S)=1$ and $g$ be a fixed non-negative integer. Then the number of algebraic families of curves on $S$ with the arithmetic genus $g$ is finite modulo Aut $(S)$. In particular, the number of non-singular rational curves on $S$ is finite modulo Aut $(S)$.

\section{Notations and Convention}

By a surface, we mean a compact complex manifold of dimension two. For a compact connected complex manifold $X$, we use the following notation.

$K_{X}$ : the canonical bundle of $X$.

$\kappa(X)$ : the Kodaira dimension of $X$. $\operatorname{Pic}(X):=H^{1}\left(X, \mathcal{O}_{X}^{*}\right)$ : the Picard group of $X$, which has the natural structure of

Communicated by K. Saito, December 8, 1989.

* Department of Mathematics, Faculty of Science, Shizuoka University, Ohya, Shizuoka, Japan. 
a commutative complex Lie group.

$\mathrm{NS}(X)$ : the Néron-Severi group of $X$, i. e. the group of algebraic equivalence classes of divisors on $X$.

$\mathcal{O}_{X}:$ the structure sheaf of $X$.

$\rho(X):=\operatorname{rank}_{\mathbf{Z}} N S(X)$ : the Picard number of $X$.

$\chi\left(\mathcal{O}_{X}\right)$ : the Euler-Poincare characteristic.

The symbol $\approx($ resp. $\sim)$ indicates algebraic equivalence (resp. linear equivalence) of divisors.

\section{$\S 1$. Preliminaries}

In this section, we shall prove Theorem $\mathrm{A}$ in the case where $S$ has the structure of the Jacobian fibration (i.e. elliptic fibration with a section). The following theorem is fundamental.

Theorem 1.1. (Miyaoka, Umezu [3]) Let $S$ be a surface with $\kappa(S) \geqq 0$ and fix a non-negative integer $g$. Then there exists a positive integer $N=N(S, g)$ which is determined only by $S$ and $g$ such that $C \cdot K_{S}<N$ for any curve $C$ on $S$ with the arithmetic genus $g$.

Proposition 1.2. Let $f: S \rightarrow \Delta$ be a relatively minimal elliptic surface over a non-singular complete curve $\Delta$ with the zero section (o) and at least one singular fiber. Assume that $\kappa(S)=1$ and $f i x$ a non-negative integer $g$. Then the number of algebraic families of curves on $S$ with the arithmetic genus $g$ is finite modulo $\operatorname{Aut}(S)$.

Corollary 1.3. Under the same conditions as in Proposition 1.2, if the Mordell-Weil rank of $S$ vanishes, the number of algebraic families of curves on $S$ with the fixed arithmetic genus $g$ is finite.

Proof of Proposition 1.2. Fix a non-negative integer $g$ and take an arbitrary algebraic curve $C$ on $S$ with the arithmetic genus $g$. Since all sections are mapped to the zero section (o) by an automorphism of $S$ and each irreducible component of a reducible singular fiber is a non-singular rational curve with self-intersection number -2 , we may assume that $C$ is a multi-section, that is, $\left.f\right|_{C}: C \rightarrow \Delta$ is a finite covering of degree $m>1$.

Now let $r$ be the rank of the Mordell-Weil group $\mathcal{E}(K)$ and take $r$ generators $\mathrm{s}_{1}, \cdots, \mathrm{s}_{r}$ of $\mathcal{E}(K)$ modulo the torsion group $\mathcal{E}(K)_{\text {tor }} . \mathcal{E}(K)_{t_{\text {or }}}$ is generated by at most two elements $t_{1}, t_{2}$ of order $e_{1}, e_{2}$ with $1 \leqq e_{2}, e_{2}\left|e_{1} ;\right| \mathcal{E}(K)_{t o r} \mid=e_{1} e_{2}$. Let $\left\{F_{\lambda}\right\}_{\lambda \in \Delta}$ (resp. $F$ ) be the set of all singular fibers of $f: S \rightarrow \Delta$ (resp. generic fiber of $f)$ and for each $\lambda \in \Delta$ we denote by $\Theta_{\lambda, i}\left(0 \leqq i \leqq m_{\lambda}-1\right)$ the irreducible component of $F_{\lambda}, m_{\lambda}$ being the number of irreducible components. Then we have 


$$
F_{\lambda}=\theta_{\lambda, 0}+\sum_{\lambda} \sum_{i \geqq 1} \mu_{\lambda, \imath} \Theta_{\lambda, \imath}, \quad \mu_{\lambda, i} \geqq 0,
$$

where $\Theta_{\lambda, 0}$ is the unique component of $F_{\lambda}$ intersecting the zero section (o). By a theorem of Shioda [5], $C$ can be written uniquely in the following way:

$$
C \approx \alpha(o)+\beta F+\sum_{i=1}^{r} a_{\imath} s_{\imath}+\sum_{j=1}^{2} b_{\jmath} t_{\jmath}+\sum_{\lambda} \sum_{k=1}^{m_{\lambda^{-1}}} d_{\lambda, k} \Theta_{\lambda, k},
$$

$0 \leqq b_{j}<e_{j}$, where $\alpha, \beta, a_{\imath}, b_{j}$ and $d_{\lambda, k}$ are all rational integers.

Claim 1. $m:=(C, F)$ is bounded above by a constant $A=A(S, g)$, which is determined only by $S$ and $g$.

Proof of Claim 1. By the canonical bundle formula of Kodaira [2], we have $K_{S} \approx\left(2 \pi(\Delta)-2+\chi\left(\Theta_{S}\right)\right) F$. Since $\kappa(S)=1$, we have $2 \pi(\Delta)-2+\chi\left(\Theta_{S}\right)>0$. Moreover by Theorem 1.1, there exists a constant $N=N(S, g)$ depending only on $S$ and $g$ such that $C \cdot K_{S}<N$. Put $A(S, g)=\frac{N(S, g)}{2 \pi(\Delta)-2+\chi\left(\mathcal{O}_{S}\right)}$. Since $C \cdot F=m$, we have $m<A$.

q.e.d.

Claim 2. In the above situation, by translating $C$ by a suitable automorphism $\Phi$ of $S$, we may assume that $0 \leqq a_{i}<m$ for all $1 \leqq i \leqq r$.

Proof of Claim 2. We can consider $S$ as a one-dimensional abelian variety $\mathcal{E}$ over the function field $K$ of $\Delta$, given with a $K$-rational point $o$. Then each section $s_{i}(1 \leqq i \leqq r)$ defines a $K$-rational point $\tilde{s}_{i}$ and let $\sigma_{i}$ be a birational mapping of $S$ induced from a translation of $\mathcal{E}$ by $\tilde{s}_{i}$ over $K$. Since $S$ is relatively minimal, $\sigma_{i}$ is an analytic automorphism of $S$. We choose integers $c_{i}$ and $\delta_{\imath}$ with $a_{\imath}=m c_{i}+\delta_{\imath}, 0 \leqq \delta_{i}<m$ and define an automorphism $\Phi$ of $S$ by $\Phi=$ $\left(\sigma_{1}^{-1}\right)^{c_{1}} \cdots \circ\left(\sigma_{r}^{-1}\right)^{c_{r}}$. Put $\bar{C}=\Phi(C)$. Clearly $\bar{C}$ is also a $m$-section of $S$, where we have

$$
m=C \cdot F=\alpha+\sum_{i=1}^{r} a_{\imath}+\sum_{j=1}^{2} b_{\jmath} .
$$

Put $C^{\prime}:=\left(\alpha+m \sum_{i=1}^{r} c_{i}\right)(o)+\beta^{\prime} F+\sum_{i=1}^{r} \delta_{i} s_{i}+\sum_{j=1}^{2} b_{\jmath} t_{\jmath}+\sum_{\lambda} \sum_{k=1}^{m \lambda^{-1}} d_{\lambda, k} \Theta_{\lambda, k}$. The divisor $\bar{C}$ (resp. $C^{\prime}$ ) cuts out on the generic fiber $F$ divisors of degree $m$ and the sum $\boldsymbol{S}(\bar{C})$ (resp. $S\left(C^{\prime}\right)$ ) of points in $\bar{C}$ (resp. $C^{\prime}$ ) under the group operation on the abelian group $F$ gives a $K$-rational point. By our construction, we get $\boldsymbol{S}(\bar{C})=\boldsymbol{S}\left(C^{\prime}\right)$. With the aid of Abel's theorem on an elliptic curve, the divisor $\left.\bar{C}\right|_{F}$ is linearly equivalent to $\left.C^{\prime}\right|_{F}$ on $F$. Hence the divisor $\bar{C}-C^{\prime}$ is linearly equivalent to a divisor contained in fibers of $f$ and $\bar{C}$ can be written in the form:

$$
\bar{C} \approx\left(\alpha+m \sum_{i=1}^{r} c_{i}\right)(o)+\bar{\beta} F+\sum_{i=1}^{r} \delta_{i} s_{i}+\sum_{j=1}^{2} b_{j} t_{j}+\sum_{\lambda} \sum_{k \geqq 1} d_{\lambda, k}^{\prime} \Theta_{\lambda, k},
$$

where $0 \leqq \delta_{i}<m$ for all $1 \leqq i \leqq r$.

q.e.d. 
Now, we continue the proof of Proposition 1.2. Since $m=C \cdot F=\alpha+$ $\sum_{i=1}^{r} a_{i}+\sum_{j=1}^{2} b_{j}$, it follows from Claim 1 and Claim 2 that $\alpha, a_{i}(1 \leqq i \leqq r)$ and $b_{j}(1 \leqq$ $j \leqq 2$ ) are all bounded by constants determined only by $S$ and $g$. Since $C$ is a multi-section, we have $0 \leqq C \cdot \Theta_{\lambda, k} \leqq C \cdot F_{\lambda}=C \cdot F=m<A(S, g)$ for each $\lambda$ and $1 \leqq k \leqq m_{\lambda}-1$. We have

$$
\left(C, \Theta_{\lambda, p}\right)=\sum_{i=1}^{r} a_{i}\left(s_{i}, \Theta_{\lambda, p}\right)+\sum_{j=1}^{2} b_{j}\left(t_{j}, \Theta_{\lambda, p}\right)+\sum_{k=1}^{m} d_{\lambda, k}\left(\Theta_{\lambda, k}, \Theta_{\lambda, p}\right) .
$$

Since the intersection matrix $\left(\left(\Theta_{\lambda, k}, \Theta_{\lambda, p}\right)\right)_{1 \leqq k, p_{\varliminf_{\lambda}-1}}$ is negative definite and $\left(C, \Theta_{\lambda, p}\right), a_{i}$ and $b_{j}$ are bounded by constants determined only by $S$ and $g$, it follows from $(*)$ that $d_{\lambda, k}$ 's are uniquely determined and bounded by constants depending on $S$ and $g$. Setting $\Gamma=\alpha(o)+\sum_{i=1}^{r} a_{i} s_{\imath}+\sum_{j=1}^{2} b_{j} t_{j}+\sum_{\lambda} \sum_{k \geqq 1} d_{\lambda, k} \Theta_{\lambda, k}$, we have $(\Gamma, F)=m$ and $C^{2}=(\Gamma+\beta F)^{2}=\Gamma^{2}+2 \beta m$. Since $\left(C, K_{S}\right)$ and $\Gamma^{2}$ are bounded by constants determined by $S$ and $g$, we infer from the above equality that $\beta$ is also bounded by constants determined only by $S$ and $g$. q.e.d.

\section{§2. Proof of Theorem A}

In this section, we shall prove Theorem $\mathrm{A}$ in the case where $S$ has the structure of an elliptic surface with multiple fibers. First, we need the following lemma.

Lemma 2.1. Let $g: S \rightarrow C$ be a relatively minimal algebraic elliptic surface with multiple fibers. Then there exists a curve $s_{0}$ with $g\left(s_{0}\right)=C$ such that $\left(s_{0}, F\right)$ divides $(D, F)$ for general fiber $F$ of $g$ and for all curve $D$ with $g(D)=C$.

Proof. Put $m_{0}=\min \{D \cdot F \mid D$ is a curve on $S$ with $g(D)=C\}$ and fix a curve $s_{0}$ with $s_{0} \cdot F=m_{0}$ and $g\left(s_{0}\right)=C$ arbitrarily. For any curve $D$ on $S$ with $g(D)=C$, we choose integers $t, r$ with $D \cdot F=m_{0} t+r, 0 \leqq r<m_{0}$. Then $0 \leqq\left(D-m_{0} s_{0}\right.$, $F)=r<m_{0}$ and $g_{*} \Theta_{S}\left(D-m_{0} s_{0}\right)$ is a coherent $\mathcal{O}_{C}$-module of rank $r$. By Serre's theorem, we have $H^{0}\left(C, g_{*} \mathcal{O}_{S}\left(D-m_{0} s_{0}\right) \otimes \mathcal{O}_{C}(n p)\right) \neq 0, p \in C$ and hence $H^{0}\left(S, \mathcal{O}_{S}(D\right.$ $\left.\left.-m_{0} s_{0}+n S_{p}\right)\right) \neq 0, S_{p}=g^{-1}(p)$ for a sufficiently positive integer $n$. A general member $\Delta \in\left|D-m_{0} s_{0}+n S_{p}\right|$ can be written in the form : $\Delta=H+\Gamma$, where $H$ and $\Gamma$ are effective divisors with $g(\operatorname{supp}(H))=C$ and $\operatorname{supp}(\Gamma)$ contained in fibers of g. Then $0 \leqq r=(\Delta, F)=(H, F)<m_{0}$ and from the minimality of $\left(s_{0}, F\right)$, we have $H=0$ and $r=0$.

q.e.d.

Now, we are ready to prove our main Theorem A.

Proof of Theorem A. Clearly, we may assume that $S$ is algebraic. Since $\kappa(S)=1, S$ has the unique structure of an elliptic surface $f: S \rightarrow C$ with multiple fibers $m_{i} E_{i}$ of multiplicity $m_{i}$ at $p_{i} \in C .(1 \leqq i \leqq l)$. Then by Kodaira [2], $S$ can 
be obtained from the basic member $g: B \rightarrow C$ by twisting and successive logarithmic transformations, that is, $S=L_{p_{1}} \cdots L_{p_{l}}\left(B^{\eta}\right)$, where $B^{\eta}$ is obtained by twisting $g: B \rightarrow C$ by $\eta \in H^{1}\left(C, \mathcal{O}\left(B_{0}^{\#}\right)\right)$.

First, we assume throughout that $g: B \rightarrow C$ is not smooth, i.e. there is at least one singular fiber.

Let $r$ be the rank of the Mordell-Weil group $\mathrm{MW}(B)$ of $g: B \rightarrow C$ and take $r$ generators $\mathrm{s}_{1}, \cdots, \mathrm{s}_{r}$ of $\mathrm{MW}(B)$ modulo the torsion group $\mathrm{MW}(B)_{t o r} \mathrm{MW}(B)_{t o r}$ is generated by at most two elements $t_{1}, t_{2}$ of order $e_{1}, e_{2}$ with $e_{2}\left|e_{1} ;\right| \mathrm{MW}(B)_{\text {tor }} \mid$ $=e_{1} e_{2}$.

By considering $B$ as an one-dimensional abelian variety $\mathcal{E}$ over the function field $K$ of $C$, each section $s_{i}$ (resp. $t_{j}$ ) defines a $K$-rational point $\tilde{s}_{i}$ (resp. $\tilde{t}_{j}$ ). Since $S$ is isomorphic to $B^{\eta}$ outside the multiple fibers, $\tilde{s}_{i}$ (resp. $\tilde{t}_{j}$ ) induces an automorphism of $\left.S\right|_{C \backslash_{i} p_{i}}$ by locally translating $B^{\eta}$ by $\tilde{s}_{i}$ (resp. $\tilde{t}_{j}$ ). And from the definition of logarithmic transformations, we see easily that it can be extended to an automorphism $g_{i}$ (resp. $h_{\jmath}$ ) of $S$. We fix a multi-section $D_{0}$ of $f: S \rightarrow C$ which enjoys the properties as in Lemma 2.1 and put $D_{i}=g_{\imath}\left(D_{0}\right)$, $T_{j}=h_{j}\left(D_{0}\right)$. Clearly $D_{i}$ 's and $T_{j}$ 's also enjoys the property in Lemma 2.1. Let $\left\{F_{\lambda}\right\}_{\lambda \in C}$ (resp. $F$ ) be the set of all singular fibers of $f: S \rightarrow C$ (resp. general fiber of $f$ ). For each non-multiple singular fiber $F_{\lambda}$ (resp. multiple singular fiber $m_{\lambda} E_{\lambda}$ ), choose an irreducible component $\Theta_{\lambda, 0}$ of $F_{\lambda}$ (resp. $E_{\lambda}$ ) of multiplicity one arbitrarily and fix them. Then we have

$$
F_{\lambda}=\Theta_{\lambda, 0}+\sum_{\lambda} \sum_{i \geqq 1} \mu_{\lambda, i} \Theta_{\lambda, i}, \mu_{\lambda, i}>0 \quad \text { (resp. } E_{\lambda}=\Theta_{\lambda, 0}+\sum_{i \geqq 1} \Theta_{\lambda, i} \text { ), }
$$

where we denote by $\Theta_{\lambda, i}\left(0 \leqq i \leqq n_{\lambda}-1\right)$ the irreducible component of $F_{\lambda}$ (resp. $E_{\lambda}$ ), $n_{\lambda}$ being the number of the irreducible components of $F_{\lambda}$ (resp. $E_{\lambda}$ ).

Claim 1. $\left[D_{0}\right],[F],\left[D_{\imath}\right](1 \leqq i \leqq r)$ and $\left[\Theta_{\lambda, i}\right]$ 's $\left(\lambda \in C, 1 \leqq i \leqq n_{\lambda}-1\right)$ are basis of $N S(S) \underset{\mathbf{Z}}{\otimes}$.

Proof of Claim 1. For an arbitrary divisor $D$ on $S$, put $d=(D, F), d_{0}=$ $\left(D_{0}, F\right)$. By Lemma 2.1, there exists a positive integer $k$ with $d=d_{0} k$. Since $\left(D-k D_{0}, F\right)=0$, the divisor $D-k D_{0}$ cuts out on the generic fiber $F$ a divisor $\delta$ of degree 0 . Since $S$ is isomorphic to $B^{\eta}$ outside the multiple fibers, the sum $\boldsymbol{S}(\delta)$ of points in $\delta$ under the group operation on the abelian group $F$ gives a section of $g:\left.B\right|_{C \backslash \bigcup_{i} p_{i}} \rightarrow C \backslash \bigcup_{i} p_{i}$. By Kodaira [3], it can be extended to a holomorphic section s of $g: B \rightarrow C$ over $C$, since $B$ is a basic member. By the Mordell-Weil theorem, we can write:

$$
\mathrm{s}=\sum_{i=1}^{r} a_{\imath}^{\prime} s_{i}+\sum_{j=1}^{2} b_{j}^{\prime} t_{j}, \quad \text { where } a_{i} \text { and } 0 \leqq b_{j}<e_{j} \text { are integers. }
$$

Put $D^{\prime}=\sum_{i=1}^{r} a_{\imath}^{\prime}\left(D_{i}-D_{0}\right)+\sum_{j=1}^{2} b_{j}^{\prime}\left(T_{j}-T_{0}\right)$. Since $\boldsymbol{S}\left(\left(D_{i}-D_{0}\right) \cdot F\right)=d_{0} s_{i}$ (resp. $\boldsymbol{S}\left(\left(T_{j}-\right.\right.$ 
$\left.\left.\left.D_{0}\right) \cdot F\right)=d_{0} t_{j}\right)$, we see that $\boldsymbol{S}\left(d_{0}\left(D-k D_{0}\right) \cdot F\right)=\boldsymbol{S}\left(D^{\prime} \cdot F\right)\left(\right.$ resp. $\left.S\left(e_{j}\left(T_{j}-D_{0}\right) \cdot F\right)=0\right)$ for a generic fiber $F$ of $f$.

By Abel's theorem on an elliptic curve, the divisor $\left.d_{0}\left(D-k D_{0}\right)\right|_{F}$ (resp. $\left.\left.e_{j}\left(T_{j}-D_{0}\right)\right|_{F}\right)$ is linearly equivalent to $\left.D^{\prime}\right|_{F}$ (resp. 0) on $F$. Hence the divisor $d_{0}\left(D-k D_{0}\right)-D^{\prime}$ (resp. $e_{j}\left(T_{j}-D_{0}\right)$ ) is linearly equivalent to a divisor contained in fibers of $f$. Hence we have:

$$
\operatorname{med}_{0} D \approx \operatorname{med} D_{0}+\beta F+\sum_{i=1}^{r} a_{\imath}\left(D_{\imath}-D_{0}\right)+\sum_{\lambda} \sum_{k=1}^{n} d_{\lambda, k}^{-1} d_{\lambda, k},
$$

where $m$ (resp. $e$ ) is the least common multiple of $m_{1}, \cdots, m_{l}$ (resp. $e_{1}, e_{2}$ ) and $\beta, a_{\imath}$ 's and $d_{\lambda, k}$ 's are all integers. And it is easy to see that $D_{0}, F, D_{\imath}$ 's and $\Theta_{\lambda, k}$ 's are linearly independent in $\mathrm{NS}(S) \underset{\mathbf{Z}}{\bigotimes} \mathrm{Q}$. Hence the claim follows. q.e.d.

Now, let $D$ be an arbitrary curve on $S$ with the fixed arithmetic genus $g$. We may assume that $f(D)=C$.

Claim 2. $d:=(D, F) \in \mathbb{Z}_{\geqq 0}$ is bounded above by a constant $A=A(S, g)$, which is determined only by $S$ and $g$.

Proof of Claim 2. By the canonical bundle formula of Kodaira [2],

$$
K_{S} \approx\left(2 \pi(C)-2+\chi\left(\Theta_{S}\right)\right) F+\sum_{j=1}^{l}\left(m_{j}-1\right) E_{\jmath} .
$$

Since $\kappa(S)=1$.

$$
M(S):=2 \pi(C)-2+\chi\left(\mathcal{O}_{S}\right)+\sum_{j=1}^{l} \frac{m_{\jmath}-1}{m_{j}}>0 .
$$

By Theorem 1.1, there exists a constant $N=N(S, g)$ depending only on $S$ and $g$ with $D \cdot K_{S}<N$. If we put $A=A(S, g)=\frac{N(S, g)}{M(S)}$, we have $d<A$. q.e.d.

Claim 3. By translating $D$ by a suitable automorphism $\rho$ of $S$, we may assume that $0 \leqq a_{i}<$ med for all $i$ in $(*)$, Claim 1 .

Proof of Claim 3. By the proof of Claim 2, we have:

$$
d_{0} D \approx d D_{0}+\beta^{\prime} F+\sum_{\imath=1}^{l} p_{\imath} E_{\imath}+\sum_{i=1}^{r} a_{\imath}^{\prime}\left(D_{i}-D_{0}\right)+\sum_{j=1}^{2} b_{j}^{\prime}\left(T_{\jmath}-D_{0}\right)+\sum_{\lambda} \sum_{k=1}^{n_{\lambda}-1} d_{\lambda, k}^{\prime} \Theta_{\lambda, k},
$$

where $\beta, p_{i}, a_{i}{ }^{\prime}, b_{j}{ }^{\prime}$ and $d_{\lambda_{1} k}^{\prime}$ are all integers and $0 \leqq p_{i}<m_{i}$. We choose integers $c_{\imath}$ and $\delta_{i}$ with $a_{i}^{\prime}=d c_{\imath}+\delta_{i}, 0 \leqq \delta_{i}<d$, and define an automorphism $\rho$ of $S$ by $\rho:=\left(g_{1}^{-1}\right)^{c_{1}} \cdots\left(g_{r}^{-1}\right)^{c_{r}}$. Put $\bar{D}=\rho(D)$. Then we have $(\bar{D}, F)=d$.

Next, put $D^{\prime}=d D_{0}+\beta^{\prime} F+\sum_{i=1}^{r} \delta_{2}\left(D_{2}-D_{0}\right)+\sum_{j=1}^{2} b_{j}^{\prime}\left(T_{j}-T_{0}\right)$. The divisor $D^{\prime}-d D_{0}$ (resp. $\left.d_{0}\left(\bar{D}-k D_{0}\right)\right)$ cuts out on the generic fiber $F$ a divisor $G^{\prime}$ (resp. $\bar{G}$ ) of degree 0 and the sum $S\left(G^{\prime}\right)$ (resp. $S(\bar{G})$ ) of points in $G^{\prime}$ (resp. $\bar{G}$ ) under the 
group operation gives a section of $g: B \rightarrow C$. Since $S\left(D_{\imath}-D_{0}\right)=d_{0} s_{2}$, we have:

$$
\begin{aligned}
\boldsymbol{S}(\bar{G}) & =\sum_{i=1}^{r} a_{\imath}^{\prime}\left(d_{0} s_{\imath}\right)-\sum_{\imath=1}^{r} c_{\imath}\left(d_{0} d s_{i}\right)=d_{0} \sum_{\imath=1}^{r}\left(a_{\imath}^{\prime}-c_{\imath} d\right) s_{\imath} \\
& =\sum_{i=1}^{r} \delta_{\imath}\left(d_{0} s_{\imath}\right) \\
& =\boldsymbol{S}\left(G^{\prime}\right) .
\end{aligned}
$$

Hence the divisor $d_{0} \bar{D}-D^{\prime}=\left(d_{0} \bar{D}-d D_{0}\right)-\left(D^{\prime}-d D_{0}\right)$ is algebraically equivalent to a linear combination of $F, E_{i}$ 's and $\Theta_{\lambda, k}$ 's over $\mathbf{Z}$. Since $0 \leqq \delta_{i}<d$, Claim 3 follows immediately by the same argument as in the proof of Claim 1. q.e.d.

Now, we continue the proof of Theorem A. We use the same notation as before. By Claim 2 and Claim 3, we have

$$
0 \leqq a_{\imath}<m e A(S, g) \quad \text { for all } 1 \leqq i \leqq r .
$$

Since $D_{0}$ and $D$ are multi-sections of $f$, we have:

$$
\begin{aligned}
& 0 \leqq\left(D, \Theta_{\lambda, p}\right) \leqq\left(D, F_{\lambda}\right)=(D, F)=d<A(S, g) \quad \text { and } \\
& 0 \leqq\left(D_{0}, \Theta_{\lambda, p}\right) \leqq\left(D_{0}, F_{\lambda}\right)=\left(D_{0}, F\right)=d_{0}
\end{aligned}
$$

for each $\lambda$ and $1 \leqq k \leqq n_{\lambda}-1$. We have:

$(* *) \operatorname{med}_{0}\left(D, \Theta_{\lambda, p}\right)=\operatorname{med}\left(D_{0}, \Theta_{\lambda, p}\right)+\sum_{i=1}^{r} a_{i}\left(D_{\imath}-D_{0}, \Theta_{\lambda, p}\right)+\sum_{k=1}^{n \lambda^{-1}} d_{\lambda, k}\left(\Theta_{\lambda, k}, \Theta_{\lambda, p}\right)$, since the intersection matrix $\left(\Theta_{\lambda, k}, \Theta_{\lambda, p}\right)_{1 \leqq k, p n_{\lambda-1}}$ is negative definite and $\left(D, \Theta_{\lambda, p}\right),\left(D_{0}, \Theta_{\lambda, p}\right)$ and $a_{\imath}$ 's $(1 \leqq i \leqq r)$ are all bounded by constants determined only by $S$ and $g, d_{\lambda, k}$ 's are also bounded by constants determined by $S$ and $g$. Setting $\Gamma=\operatorname{med} D_{0}+\sum_{i=1}^{r} a_{\imath}\left(D_{\imath}-D_{0}\right)+\sum_{\lambda} \sum_{k=1}^{n} d_{\lambda, k} \Theta_{\lambda, k}$, we have $\left(\operatorname{med}_{0}\right)^{2} D^{2}=(\Gamma+\beta F)^{2}$ $=\Gamma^{2}+2 \beta \operatorname{med}_{0}$. Since $\left(D, K_{S}\right)$ and $\Gamma^{2}$ are all bounded by constants determined by $S$ and $g$, we infer from the above equality that $\beta$ is also bounded by constants determined only by $S$ and $g$. Hence if we express $D$ as a linear combination of $D_{0}, F, D_{i}(1 \leqq i \leqq r)$ and $\Theta_{\lambda, k}\left(1 \leqq k \leqq n_{\lambda}-1\right)$ in $N S(S) \underset{\mathbf{Z}}{\otimes}$, the number of the possible values for each coefficient is bounded by $S$ and $g$ and Theorem A has been proved.

Next, we consider the case where $f: S \rightarrow C$ is a Seifert fiber space, that is, $S$ is an elliptic surface with constant moduli which has at most multiple singular fibers. Let $D$ be an arbitrary curve with the fixed arithmetic genus $g$. We may assume that $f(D)=C$. Then by completely the same method as above, we can show that by translating $D$ by a suitable automorphism $\rho$ of $S$, we may assume that:

$m D \approx \alpha D_{0}+\beta F$, where $m$ is the least common multiple of $m_{\imath}$ 's and $\alpha$ and $\beta$ 
are integers. Hence we can easily show the boundedness of $\alpha$ and $\beta$ by Miyaoka's Theorem 1.1.

Thus we have finished the proof of Theorem A.

\section{References}

[1] Bloch, S., Kas, A. and Lieberman, D., Zero cycles on surfaces with $p_{g}=0$, Compositio Math., 33 (1976), 135-145.

[2] Kodaira, K., On the structure of compact analytic surfaces, II-III, Amer. J. Math. 86 (1964), 751-798, 88 (1966), 682-721.

[3] Miyaoka, Y. and Umezu, Y., Boundedness of the curves of a fixed genus on a surface of general type, preprint.

[4] Namikawa, Y., Finiteness of numbers of curves on a surface, Symposium on algebraic geometry at Kinosaki, (1985), 1-5. (in Japanese)

[5] Shioda, T., On elliptic modular surfaces, J. Math. Soc. Japan, 24 (1972), 20-59. 